\section{European researchers encourage improvement in lab animal welfare}

Sir- Rodents are currently excluded from the protective regulations of the US Animal Welfare Act. Last September the US Department of Agriculture (USDA) decided to take steps to include rats, mice and birds in the act. This decision was heavily criticized by the Association of American Medical Colleges, the National Association of Biomedical Research and the Federation of American Societies for Experimental Biology ${ }^{1,2}$. Yet the USDA is determined to implement its decision and is planning to make it effective by October.

The opposition by these leading science organizations in the United States is not supported by the American Association for Laboratory Animal Science and also contrasts with European perspectives on the issue. Last September, the European Science Foundation (ESF) published a position paper on the use of animals in research ${ }^{3}$. The guidelines from this paper, summarized below, were adopted by the ESF assembly at the end of November.

The ESF consists of 67 leading sciencefunding agencies, research councils and academies of science from 23 European countries. Its role is to stimulate cooperation between national organizations and individual scientists from different countries and to advise on science policy. Its position paper states the conditions that must be met to make the use of animals for research purposes morally acceptable. The ESF encourages scientific organizations and individuals involved in animal experimentation to follow these guidelines, which can be summarized as follows:

- Laboratory animals not only have an instrumental value, but also an intrinsic value, which must be respected.

- While accepting the need for animals to be used to advance scientific knowledge and to promote human and animal health and well-being, the ESF strongly endorses the reduction, replacement and refinement principles.

- Research to improve the welfare of animals should be encouraged and actively supported.

- Before a programme of research, the proposed animal use should be evaluated independently, including an assessment of the likely benefit and suffering.

- Investigators should assume that procedures that would cause pain in humans also cause pain in other vertebrates, unless there is evidence to the contrary. - Investigators should be adequately educated and trained through accredited courses on laboratory-animal science, including discussion of animal alternatives, welfare and ethics.

- The ESF encourages journals to include in their publication policy a statement on the ethical use of animals.

The ESF recognizes the important role of journals in the ethical use of animals in research. It is, therefore, unfortunate that most journals publishing in this area do not have stated guidelines that must be a prerequisite for consideration of manuscripts ${ }^{4}$. A recent study shows that only 13 of 83 relevant journals contain a comprehensive statement on the ethical use of animals. In 42 journals (59\%) there was no statement at all ${ }^{5}$.

The ESF guidelines demonstrate that the scientific community in Europe is leaving behind its defensive attitude, and is more proactive in expressing what it sees as standards to adhere to when it comes to the use of animals in research. This should be a benefit for animals and for science.

\section{Bert van Zutphen}

Department of Laboratory Animal Science, Faculty of Veterinary Medicine, Utrecht University, PO Box 80.166, 3508TD Utrecht, The Netherlands

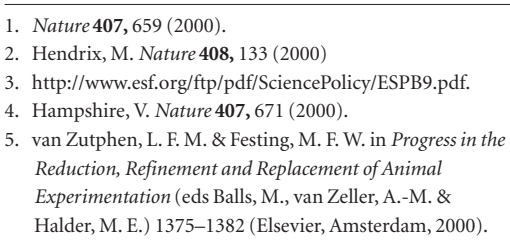

\section{Users must help to keep public databases correct}

Sir - With the continued growth of the public DNA sequence databases, and the recent addition of the $11,000,000,000$ th nucleotide to GenBank (including DDBJ, EMBL and GenBank), it is timely to assess how we use these databases.

GenBank is the archive of all publicly available DNA, RNA and protein sequences. Upon publication a new sequence and its annotations appear in it. Investigators use GenBank in many ways, most commonly for similarity searches such as BLAST; to retrieve records; and for sequence analysis, multiple sequence alignment or pattern finding search. Errors sometimes occur in GenBank, ranging from the trivial (incorrect postal codes), to the misleading (30 nucleotides of vector left on the ends of a record), to the mission-critical (a full length mRNA without a coding sequence (CDS) annotated on it). Also very common are incomplete references that prevent researchers from linking the GenBank record to the publication that refers to it first.

Over the years some people have chosen to report these errors, but in most cases they are left unmodified. An uncorrected 'discovered' error is one of the worst possible failings in GenBank, so if you discover an error, report it to the database (update@ncbi.nlm.nih.gov) and it should be rectified - although a follow-up is advised to make sure this gets done.

If you are a submitter, look at the record you submitted a few years ago: is it still correct? Was the citation ever updated? Take pride in the sequences that carry your name! Our ability to interpret genomes depends on all of these records being as accurate as possible. This is a task for all users of the databases.

Francis Ouellette

Bioinformatics Core Facility, Centre for Molecular Medicine and Therapeutics, University of British Columbia, 950 West 28th Avenue, Vancouver, British Columbia V5Z 4H4, Canada

\section{The long-term answer: fight fire with research}

Sir - We agree with your Editorial (Nature $406,661 ; 2000)$ in that the spectacular fires of last year should be a stimulus for improving management of forest ecosystems in the American west. In fact, through the leadership of the US Department of Agriculture Forest Service, a new programme is being implemented to improve forest management, in view of fire and other hazards that endanger forest health.

However, we strongly disagree with your statement that the Forest Service "uses its money to pay its staff, not to conduct high-quality research into forest management".

In reality the Forest Service, beginning in the 1920s under Rafael Zon, has exerted global leadership in forestry research. The ecosystem models used to manage fires in the west and to accomplish appropriate restoration are products of long-term ecological research, started decades before the value of such research was realized in the academic community or other scientific organizations. Five of the National Science Foundation long-term ecological research sites are Forest Service research sites, where such work has been taking place for many years.

Forest Service research is cost-effective. About $7 \%$ of the agency's budget, some $\$ 220$ million a year, supports six research stations, the Forest Products Laboratory and the International Institute of Tropical Forestry.

Thomas J. Mills ${ }^{\star}$, Ariel E. Lugo $\dagger$

${ }^{*}$ Pacific Northwest Research Station, PO Box 3890 , Portland, Oregon 97208, USA

†International Institute of Tropical Forestry, PO Box 25000, Rio Piedras, Puerto Rico 00928-5000 